\title{
CONSIDERACIONES SOBRE EMPLEABILIDAD EN EDUCACIÓN SUPERIOR
}

\section{Natalia Orellana ${ }^{1}$}

\begin{abstract}
RESUMEN
Este artículo provee una revisión de los aspectos centrales de los debates en torno a la relación entre la educación superior y el mundo del trabajo, con el propósito de contextualizar la emergencia de la empleabilidad como concepto, práctica e instrumento de política en educación superior. En este ámbito se observa que el alcance masivo y eficaz de su difusión internacional se contradice con un despliegue altamente criticado, a partir de la naturaleza de los dispositivos que condicionan su interpretación y la falta de diálogo interdisciplinar en su tratamiento. Se concluye que empleabilidad, en este contexto, tiende a presentarse como una instrumentalización de la rendición de cuentas respecto de los procesos de enseñanza-aprendizaje. Esto, a partir de bases conceptualmente débiles que dan lugar a su configuración problemática, disociadas de la investigación educativa, específicamente, del aporte de la teoría de carrera, la educación para la carrera y la orientación profesional que brindan contenido al desarrollo de habilidades para la gestión de la carrera (o empleabilidad) desde la perspectiva de proceso formativo.
\end{abstract}

Palabras clave: desarrollo de carrera, educación superior, empleabilidad, mundo del trabajo, teoría de carrera.

\section{CONSIDERATIONS ABOUT EMPLOYABILITY IN HIGHER EDUCATION}

\begin{abstract}
This paper provides a review of central features on the debates about the relationship between higher education and the labour market with the aim to contextualize the emergence of employability as a concept, practice, and policy in higher education. In this environment, despite its extensive international diffusion, it suffers significant and criticized contradictions regarding its appropriation, due to the presence of multiple interpretations and the lack of interdisciplinary dialogue to approach it. Thus, in this context, employability tends to be presented as an accountability tool for the teaching and learning process, due to conceptually weak bases completely unrelated to educational research, that give way to a problematic configuration. Its conceptualization does not take into account, for example, contributions from career theory, career education, and career orientation for the development of career management (or employability) skills, from a process-oriented perspective.
\end{abstract}

Keywords: career development, career theory, employability, higher education, world of work.

Facultad de Letras, Pontificia Universidad Católica de Chile, Santiago, Chile. Contacto: norellanag@uc.cl 


\section{Introducción}

En su evolución histórica, la educación superior se ha ido configurando a través de diversas formas de acción social caracterizadas por principios que se mantienen hasta nuestros días, como son: la enseñanza de conocimientos innovadores para su tiempo y el desarrollo de nuevo conocimiento para profundizar el entendimiento acerca de la naturaleza, la sociedad y la humanidad, así como también para mejorar la sociedad (Shin, 2014). En este proceso, el valor de uso del conocimiento se ha constituido como un aspecto transversal a los distintos momentos de este fenómeno macrosocial que, a pesar de sus diversos propósitos y formas organizacionales, ha mantenido la estabilidad de ciertos atributos centrales que le caracterizan como un campo de acción social, determinado por su vínculo con las condiciones y el contexto histórico de su establecimiento y las influencias de demandas sociales y productivas de cada periodo.

Desde sus inicios, la universidad moderna — proveniente de la Europa medieval del siglo XII y el subsecuente establecimiento de universidades en torno al 1800 — se ha legitimado por el valor de uso que la sociedad ha dado a su quehacer. Desde su génesis, cuando las universidades consistían en escuelas especializadas (conocidas como studium generale), su principal factor de éxito fue la satisfacción de las expectativas profesionales de la sociedad de su día, a través de la formación de teólogos, juristas, físicos y académicos (Pedersen, 1997) y, posteriormente, cubriendo las aspiraciones humanísticas del periodo pos-Renacimiento (Brubacher, 1984).

\section{Educación superior y mundo del trabajo}

La relación de la educación superior con su entorno social y productivo evidencia el permanente esfuerzo que, a lo largo de su historia, las universidades han debido desplegar para sobrellevar la

2 Referencia basada en la definición de campos de Bourdieu (1997) como microcosmos sociales dinámicos, cambiantes, reconocidos por sus cualidades relacionales y dialécticas. Compuestos por actores — dominados y dominantes — en disputa de poder por los bienes específicos poseídos y/o producidos por el campo, por el monopolio sobre los mecanismos de reproducción y tipo de poder efectivo en él, funcionando como mercados (citado en Brown \& Baker, 2007). 
tensión entre sus propias fuerzas internas de desarrollo y las fuerzas externas de oferta y demanda, tanto para su subsistencia como para su estabilidad e institucionalización (Brunner, 2009). En consecuencia, abordar la relación entre educación superior y mundo del trabajo evoca aspectos centrales de estos campos, tales como la posición y el significado que cada uno de ellos representan en la sociedad. Se trata, entonces, de un ejercicio de reflexión en torno a diversos entendimientos sobre la naturaleza del conocimiento y su valor de uso pragmático y de intercambio (Barnett, 2000).

Esta relación ha sido mediada por connotaciones históricamente en disputa de hegemonía, asociadas a la significancia de acciones humanas de grupos dominantes y dominados que, desde el centro de estructuras sociales antiguas, han arraigado, heredado y transmitido ideas acerca de su naturaleza que se evidencian hasta nuestros días. Por tanto, comprenden imaginarios fundacionales en torno a la educación superior y el trabajo. Para la primera se asocian ideas como el poder, la libertad, el ocio, lo elevado y puro de la búsqueda desinteresada de conocimiento (valor intrínseco). La segunda, por su parte, se construye sobre la evolución histórica de la acción humana del trabajo; esto es, desde la subordinación y la alienación, como lo mundano e innoble de la actividad laboral.

En el centro de las discusiones en torno a la relación entre la educación superior y el mundo del trabajo subyace el debate acerca del rol de la primera en la sociedad. Entonces, se observa que la idea de la educación superior y sus diversas perspectivas es frecuentemente planteada en términos dicotómicos: evolución/involución, crisis/ apogeo, unidad/fraccionamiento, anticuado/nuevo (Barnett, 1990; Ben-David y Zloczower, 1962; Brubacher, 1984; Kerr, 1962; Scott, 1984; Shin, 2014). Un debate filosófico respecto del conocimiento que se cristaliza de modo cíclico en los discursos académicos, públicos y cotidianos; definido como tal, por la valoración simbólica que portan sus concepciones idealizadas, sobre las que se construyen y deconstruyen las ideas acerca de sí misma, que también son ideas acerca de las sociedades donde se desarrollan en un determinado contexto. 
Estas aproximaciones se expresan a través del lenguaje que, como un modo de acción social, se encuentra históricamente situado en una relación dialéctica con otras facetas de lo social (Fairclough, 2003). Los diversos entendimientos respecto de por qué se instaló y qué es lo que hoy conocemos como educación superior remiten al para qué de su quehacer. Y es justamente en la conceptualización de sus funciones donde se efectúa este ejercicio de retorno a su configuración conceptual y, aún más atrás, a sus fuerzas de impulso. Dicho proceso se relaciona con la búsqueda de sentido de este fenómeno que, durante los últimos nueve siglos, gradualmente se ha ido constituyendo como un campo altamente diferenciado, clave en la reproducción de sistemas simbólicos.

Se emplea la expresión mundo del trabajo que aparece en los aportes de Kogan y Brennan (1993) y Teichler (2009) para referir, en amplitud, al quehacer ocupacional de los individuos. Se busca contemplar tanto la sustancia como las condiciones de la acción humana que produce o modifica algo externo de sí misma, implicando cierta racionalidad a su acción. Por tanto, al término se le atribuye una multidimensionalidad intrínseca. Con su utilización, se pretende avanzar por sobre la dualidad recurrente asociada al concepto de trabajo, que Noguera (2002) identifica como la oposición valoración/ desprecio y Oliveira (2014) como sufrimiento/disfrute. Esta noción se adopta para superar la visión de trabajo inserta en el mercado como factor de producción de la economía formal, vinculada esencialmente al nexo salarial. Entonces, la conceptualización mundo del trabajo con la cual la educación superior aparece condicionada en este planteamiento, pone en un mismo plano de análisis dos aspectos de la vida social que tienden a ser asociados como campos de la acción humana en disputa.

Un modo de graficar esta situación es hacer un paralelo entre las ideas contenidas en visiones dominantes diversas respecto del conocimiento y la universidad. Como punto de partida, los escritos del Cardenal John H. Newman (1852/2016) son expresión del énfasis en el valor del conocimiento y su búsqueda como una acción humana con valor en sí misma; mientras que los postulados de Lyotard (1984) expresan críticamente la sobrevaloración contemporánea 
del conocimiento instrumental, en lo que denomina ideología del desempeño. En Newman la acción está asociada a una dimensión estético-expresiva, propia de la autorrealización personal, por tanto, de valor intrínseco. A su vez, en Lyotard se apunta a una dimensión cognitivo-instrumental, de valor extrínseco ${ }^{3}$, propio de la conceptualización de la actividad laboral o trabajo.

En este rango de ideas sobre el valor de conocimiento, se puede observar que tanto la universidad (cualesquiera sean sus modelos) así como la noción misma de trabajo (cualquiera sea su valoración sociocultural preponderante), son parte del cambio de paradigma moderno en donde la acción se valora como medio para el éxito, desprendiéndose de su contenido ético —valor intrínseco(Bell, 1976). Tanto así que, en nuestro tiempo, el quehacer de la universidad es considerado un trabajo, no una actividad libre ni desprendida de intereses, ni tampoco reservada a ciertos miembros especiales de la sociedad.

Durante la segunda mitad del siglo XX se consolidó una nueva relación de las universidades con la organización económica de la sociedad, como un vínculo crucial que define claramente las funciones de la universidad actual, donde la disputa en torno al valor extrínseco de uso del conocimiento corresponde a la perspectiva predominante en cuanto uso productivo del quehacer de ella. En dicho periodo, los principios conductores para garantizar el desarrollo económico se basaron en el convencimiento de la necesidad de reforzar la educación superior para incrementar la fuerza de trabajo con niveles educacionales avanzados y así responder a las demandas del crecimiento industrial (Bastalich, 2010,). Por tanto, más allá de las posturas filosóficas respecto de las funciones de la educación superior, su posicionamiento como un aspecto determinante para el empleo de los graduados se fue transformando en una de las características de la universidad moderna de niveles de acceso masivo.

Tomado de la conceptualización aportada por Noguera (2002, pp. 145-146) acerca de conceptos de trabajo en relación con las dimensiones de la acción. 


\section{Universidad y empleabilidad}

La universidad contemporánea, al inicio del siglo XXI, se situó en una posición central para la economía y sociedad del conocimiento, posexpansión, con niveles de acceso masivo avanzando hacia el acceso universal ${ }^{4}$, fuertemente interpelada por su entorno institucional (Krücken, 2011). Ella se encuentra desafiada a responder, adaptarse, proyectarse e incluso redefinirse, en consideración con demandas sociales y productivas — diversas y contradictorias—, concebidas desde la perspectiva dominante de la doctrina económica.

Más allá de la finalidad socializadora, las funciones básicas de la educación superior relacionadas con el mundo del trabajo que se han ido consolidando, de acuerdo con Teichler (2009) son las siguientes:

a. una preparadora, orientada a nutrir las capacidades cognitivas y posiblemente efectivas y sensomotrices que pueden ser útiles para hacer frente a las tareas del trabajo; $y$,

b. una distribuidora de estatus, como factor que permite o impide el acceso a ocupaciones de privilegio y provee los medios para los logros profesionales y, por ende, de remuneración y estatus socioeconómico.

En este contexto, la introducción explícita del concepto de empleabilidad en educación superior surge en el informe británico de 1997 Dearing Report, en referencia a la necesidad añadir otras competencias además del conocimiento cognitivo ${ }^{5}$ en los planes de estudio, como una vía para garantizar un mejor rendimiento en el trabajo. Aspecto que Yorke (2004) explica como un objetivo que los gobiernos alrededor del mundo han impuesto, en diversa medida, a los sistemas de educación superior nacionales. Es en este marco discursivo donde la empleabilidad es incorporada en la educación superior a través de la política pública, que recoge los lineamientos de la teoría de capital humano, y enfatiza la eficiencia de la educación

4 En referencia a los niveles de acceso en Trow (1974); élite, masivo, universal.

5 Las habilidades clave destacadas fueron: comunicación, habilidades numéricas, tecnología de la información, y aprender a aprender. 
formal para aumentar la productividad individual y el crecimiento económico (Marginson, 2015; Tomlinson, 2017).

En efecto, una agenda en torno a ella se ha establecido en sistemas de educación superior de las diversas regiones del mundo, denotando un valor extrínseco del conocimiento, el cual se ha visto reflejado en posturas normativas potenciadas por múltiples canales, entre los que destacan organizaciones internacionales ${ }^{6}$ y gobiernos nacionales (Arora, 2013). Un hito en este sentido lo marca la Conferencia Mundial de la Educación Superior de París 1998, donde la Organización de las Naciones Unidas para la Educación, la Ciencia y la Cultura (Unesco, por sus siglas en inglés) emplazó al incremento de la empleabilidad en la sociedad del conocimiento, por medio de lineamientos de política explícitos que desafiaron a las universidades a responder como actores racionales frente a las demandas sociales y productivas. La Unesco atribuyó a los proveedores de educación una función prospectiva, asociada a su deber de fomentar la creación de empleos (Unesco, 1998). Durante los años siguientes, este llamado se ha visto fortalecido por medio de la difusión internacional de mecanismos estandarizadores y de diferenciación vertical, dispuestos a través de sistemas de aseguramiento de la calidad y la instalación de rankings universitarios específicos de empleabilidad, tales como QS Graduate Employability Ranking y The Times Higher Education Global University Employability Ranking ${ }^{7}$.

En paralelo, numerosas publicaciones acerca de la historia del concepto y sus diversas interpretaciones disciplinares dan cuenta de la destacada notoriedad que ha adquirido durante los últimos 20 años, y también de su reputación de concepto vacío y engañoso (Hinchliffe, 2006; McQuaid \& Lindsay, 2005; Rothwell \& Rothwell, 2017; Teichler, 2015). Al coexistir múltiples interpretaciones diferenciadas por las perspectivas disciplinares que la abordan, "no se constata evidencia significativa de que se esté llevando a cabo una

6 Tales como OECD, World Bank, European Comission, European Centre for the Development of Vocational Training, Organización Internacional del Trabajo, Programa de las Naciones Unidas para el Desarrollo, Banco Interamericano de Desarrollo, European Lifelong Guidance Policy Network.

7 Disponibles en: qs.com y timeshighereducation.com, respectivamente. 
conversación interdisciplinaria al respecto", según constatan Artess, Hooley y Mellors-Bourne (2017, p. 39).

Dicha característica dice relación con la utilización de la empleabilidad como "slogan" (Teichler, 2015, p. 278) frente a la instrumentalización de rendición de cuentas respecto de procesos de enseñanza-aprendizaje. Esto, dentro de la instalación del énfasis contemporáneo en la existencia de atributos de los graduados que son relevantes para el trabajo y el desarrollo del proyecto vital, que suelen ser referidos como: habilidades genéricas, habilidades clave, habilidades básicas, habilidades de empleabilidad, principalmente denominadas como: competencias (Hager, 2006; Tomlinson, 2017). Entre las definiciones de empleabilidad de mayor consenso en la investigación especializada destaca:

A set of achievements —skills, understandings and personal attributes - that makes graduates more likely to gain employment and be successful in their chosen occupations, which benefits themselves, the workforce, the community and the economy (Yorke, 2004, p. 8) . $^{8}$

Teorías y métodos para el desarrollo de los atributos de empleabilidad están ampliamente contenidos en ámbitos interdisciplinares especializados en el tema, pero escasamente citados en la investigación especializada en educación superior cuando se aborda su relación con el mundo del trabajo ${ }^{9}$. Sin embargo, su posicionamiento está adquiriendo una reforzada relevancia, en cuanto el acceso a la educación superior avanza hacia niveles masivos y universales $^{10}$ en numerosos sistemas nacionales; las dinámicas de creación y destrucción de empleo se complejizan; se incrementa la diversidad del estudiantado junto con sus necesidades e intereses;

8 (Un conjunto de logros — destrezas, entendimientos y atributos personales- que hacen a los graduados más propensos a tener empleo y ser exitosos en sus ocupaciones elegidas, en beneficio de ellos mismos, de la fuerza laboral, de la comunidad y de la economía), traducción propia.

9 Con excepciones en los países en donde la educación para la carrera y la orientación profesional muestra los mayores desarrollos en investigación y práctica, como en Reino Unido, Finlandia, Canadá, Estados Unidos, Australia y España.

10 En referencia a los niveles de acceso a la educación superior categorizados en Trow (1974) como élite, masivo y universal. 
las economías y sociedades del conocimiento requieren profesionales flexibles para encontrar, mantenerse y progresar en el sistema de empleo, entre otros aspectos relevantes de contexto que posicionan la importancia de preservar el protagonismo de cada persona en su recorrido ocupacional, desde una perspectiva de activación y ampliación de sus potencialidades.

\section{El problema de la empleabilidad}

En el entorno institucional de las universidades, la configuración de la empleabilidad y los debates que esta encabeza están profundamente marcados por su peculiaridad en cuanto concepto de política pública, apropiado y puesto en acción como un discurso protegido por un manto de neutralidad. Como si se tratase de algo bueno en sí mismo, adoptado como un estado de regla general y dando por hecho su comprensión de sentido común, en términos de Meyer y Rowan (1977), es decir, institucionalizándose. Esto ha ocurrido a partir de su componente instrumental, asociado al valor extrínseco del conocimiento.

Considerando la teoría organizacional para el estudio de la educación superior, específicamente la conceptualización de mitos racionalizados aportada por Meyer y Rowan (1977) en referencia a la configuración de un conjunto de reglas institucionalizadas que las organizaciones despliegan como mecanismos para dar sentido, legitimidad y organicidad funcional a su acción (Acosta y Buendía, 2016), la institucionalización de la empleabilidad se expresaría en la configuración de un mito racionalizado, que este artículo identifica en términos de: asegurar la inversión vía incorporación de habilidades o destrezas adicionales. Pues en su proceso de adopción reproduce discursos propios del mercado capitalista, evidenciados en su aproximación predominante que caracteriza cuatro aspectos de la empleabilidad institucionalizada, tomados de las síntesis de Tomlinson (2017) y Teichler (2015):

- que la responsabilidad del empleo recae en el individuo;

- que fortalece la hegemonía capitalista de justificación del statu quo; 
- que tiende a situar las supuestas necesidades de los empleadores por sobre las de estudiantes y graduados;

- y que representa al estudiante como consumidor cuya motivación intrínseca sería la búsqueda de salario y estatus.

En el proceso de difusión de este mito racionalizado, se identifica la problemática de su puesta en acción marcada por la aproximación dominante del agente (en el contexto de la instrumentalización de la empleabilidad) desde una visión monodimensional fundada en la capacidad de los individuos para adaptarse a las demandas del mercado laboral, incluyendo inversión en tiempo, esfuerzo y/o dinero para incrementar o mejorar sus habilidades, conocimientos, competencias u otros atributos requeridos, de acuerdo con Tholen (2013).

De este modo, las funciones de la educación superior relacionadas con el mundo del trabajo han focalizado los atributos de los graduados inmediatamente útiles para la inserción laboral. La empleabilidad, así, se toma como resultado e indicador de calidad de la educación sobre el empleo de los graduados. Una instrumentalización de nivel micro, centrada en los individuos, quienes resultan responsables de su éxito o fracaso, desafiados a ser flexibles y previsores de los cambios en las dinámicas del sistema de empleo. A diferencia de las aproximaciones de carácter multidimensional —que contemplan el reconocimiento de que la empleabilidad no solo depende de capacidades y voluntades individuales, sino también de múltiples aspectos de su contexto que quedan fuera de su control— que han quedado relegadas (McQuaid \& Lindsay, 2005).

Lo anterior se relaciona con que su difusión fue invisibilizando los aportes de la investigación educativa centrados en capacitar a los individuos para tomar decisiones informadas y transiciones relacionadas con sus aprendizajes y su trabajo basados en teoría. Entre estas, destacan la teoría de carrera y el movimiento de educación para la carrera y orientación profesional, que surgen a inicios del siglo XX (Rodríguez, 2002). 
La teoría de carrera ${ }^{11}$ aborda el rango de procesos diseñados para capacitar a los individuos para que tomen decisiones informadas y transiciones relacionadas con sus aprendizajes y su trabajo (Arulmani, Bakshi, Leong \& Watts, 2014). En ese marco, el movimiento de educación para la carrera brinda contenido teórico y metodológico en lo que respecta a la formación y el desarrollo estudiantil integrador de capacidades, atributos y aprendizajes para la planificación del proyecto vital, considerando las posibilidades de desempeño ocupacional desde una perspectiva amplia (carrera). A la vez que la orientación profesional adquiere una gran relevancia para facilitar el que las personas reconozcan sus capacidades e intereses, tomen decisiones formativas y de empleo, y gestionen el propio aprendizaje a lo largo de la vida, así como otros procesos en los que se ponen en juego las propias competencias (Álvarez y Bisquerra, 2012). Desde esta perspectiva se habla de empleabilidad como desarrollo de habilidades para la gestión de la carrera, pues se trata de planteamientos orientados a crear un fuerte vínculo entre la actividad formativa y la vida activa, que prepare para el trabajo y estimule el desarrollo de la carrera (McCarthy, 2016).

El principal ejemplo acerca del efecto de invisibilización de dichos aportes se observa en el uso simplista y recurrente de la empleabilidad como sinónimo de empleo, principalmente en las agendas políticas y mediáticas (Arora, 2013; Burke, Scurry, Blenkinssop \& Katy, 2017). La empleabilidad se ha posicionado como referente conceptual de la relación entre educación superior y mundo del trabajo desde una perspectiva que deja fuera el atributo clave de ese vínculo: su multidimensionalidad, al no diferenciar empleo (estado ocupacional) de empleabilidad (herramientas y preparación) para las transiciones educacionales y laborales. Al tiempo en que en una importante cantidad de literatura, esta se interpreta desde la perspectiva de la demanda y, por tanto, como una requerida subordinación de la universidad a los requerimientos del sector productivo, tal como observan Scheele y Brunner (2009).

11 Tanto la teoría de carrera como el movimiento de educación para la carrera y la orientación profesional son referidos internacionalmente como desarrollo de carrera (International Centre for Career Development and Public Policy, ICCDPP, 2017). 
Otro ejemplo donde la empleabilidad en la educación superior se ha posicionado sobre la tendencia de no informarse ni construirse a partir de la investigación del propio campo, se observa en el hecho de que las experiencias asociadas presentan un significativo nivel de incongruencia entre la teoría y la práctica. Esto, porque en su diseño y ejecución tienden a no considerar las metodologías de enseñanzaaprendizaje pertinentes para la transferencia de los conocimientos, las destrezas y las habilidades relacionadas con el fortalecimiento de las herramientas de empleabilidad, lo que se traduce en prácticas con mayor énfasis informativo que formativo, de acuerdo con el estudio Hager (2006).

A la luz de la discusión acerca de las problemáticas sintetizadas anteriormente, la literatura de la última década evidencia un giro desde las directrices predominantes de la teoría del capital humano hacia un entendimiento más complejo en torno a esta relación, debido a la integración de los aportes de diversas disciplinas tales como la psicología, la educación, la sociología y la ciencia política, según constatan las revisiones de Artess et al. 2017; Coetzee, 2014; Holmes, 2013; y Valiente, 2014. Un vuelco que desafía la subordinación de la universidad a las demandas de los sistemas de empleo y acentúa la multidimensionalidad de los aspectos involucrados.

Existe consenso respecto de que la empleabilidad requiere un tratamiento intencionado en el proceso formativo, que recoja la evidencia e investigación especializada al respecto (Burke et al., 2017). Destaca la relevancia de la infusión curricular, esto es, la integración del desarrollo de habilidades para la gestión de la carrera o empleabilidad a nivel curricular, además del extracurricular y cocurricular (Artess et al., 2017; Rodríguez, 2002); en específico, el desarrollo de recursos psicosociales, entendidos como aquellas capacidades (tales como motivación personal, flexibilidad, actitud proteica, adaptabilidad e identidad de carrera) que ayudan a las personas a influir de manera efectiva en el entorno y a regular su comportamiento. Por lo cual, la autogestión o autorregulación se ubica como una faceta integral de la empleabilidad (Coetzee, 2014). 
Los modelos de empleabilidad basados en este enfoque que destacan por su influencia son: DOTS Análisis (Law \& Watts, 1977), USEM (Knight y Yorke, 2003) y CareerEDGE (Dacre Pool \& Sewell, 2007). Todos ellos aportan una visión centrada en el desarrollo de la persona desde una concepción integrada del aprendizaje, desplegada a través de programas que, de acuerdo con Rodríguez (2002) contemplan:

- orientación personalizadora ligada al conocimiento de sí mismo;

- orientación profesionalizadora, relacionada con el conocimiento acerca del trabajo;

- orientación exploratoria, vinculada al desarrollo de destrezas para planificar y tomar decisiones;

- además de un currículo activador, a través de acciones dispuestas para conocer el mundo ocupacional.

Los países con mayores avances en esta materia (tanto desde las políticas públicas de trabajo y educación como dentro de la gestión universitaria) son Reino Unido, Canadá, Finlandia, Dinamarca, Estados Unidos, Australia, Nueva Zelanda, España, Corea del Sur y Japón ${ }^{12}$.

Chile se encuentra en una situación precaria al respecto, dada por la ausencia de trabajo articulado entre los ministerios relacionados, la falta de lineamientos de política acerca de la materia, y una perspectiva expresada en dos focos: intermediación laboral (Ministerio del Trabajo y Previsión Social) y elección vocacional (Ministerio de Educación), que constituyen una aproximación limitada que no contempla los aportes de la educación para la carrera y orientación profesional frente a las transformaciones derivadas del acceso masivo a la educación superior y los desafíos del mundo del trabajo del siglo XXI, que complejizan las trayectorias profesionales con inusitada rapidez, de acuerdo con el reporte 2017 respecto del desarrollo de carrera en Chile (Orellana \& Serhati, 2017).

12 Reportes país disponibles en el sitio web del International Centre for Career Development and Public Policy: iccdpp.org 
En casos como el chileno, esta situación implica una presión adicional a las universidades, desafiadas a:

a. abordar la empleabilidad como respuesta a la expresión dominante de nivel de sistema que la operacionaliza como indicador de calidad, a partir de su interpretación como sinónimo de empleo, ligada al salario, estatus ocupacional y tiempo de inserción laboral. Explícita y formalmente instalada en criterios de evaluación de carreras y proveedores, por parte de los sistemas de acreditación nacional (Zapata y Clasing, 2016) y de información sobre educación superior del Ministerio de Educación (SIES, 2018); y

b. conciliar ese tipo de respuesta con aquellas (en diversa medida instintivas y/o racionales) que derivan de la naturaleza de su quehacer y desarrollo orgánico, por tanto, que pueden involucrar también procesos formativos, desarrollo estudiantil y docente.

Este es un contexto propicio para que la empleabilidad tienda a ser referida como si se tratara de un asunto vinculado únicamente a los graduados y/o los estudiantes que se encuentran en las etapas finales de sus estudios (énfasis en los resultados), disociada de su tratamiento desde la experiencia formativa (énfasis en el proceso) que pone en el centro la permanente adquisición de destrezas, habilidades y atributos para la gestión del proyecto vital de todos los estudiantes.

\section{Conclusiones}

A partir de la difusión internacional de la empleabilidad en educación superior, su relación histórica con el mundo del trabajo ha sido contenida y, por tanto, dominada por esta referencia configurada en bases conceptuales de la doctrina económica que han demostrado ser insuficientes para su aplicación comprehensiva.

En el centro de esta problemática se ubica su instrumentalización como discurso de política pública, que este artículo grafica como la instalación de un mito racionalizado consistente en asegurar la inversión vía incorporación de habilidades y destrezas adicionales. 
Sustentado en la difusión internacional de una agenda de empleabilidad que — a pesar de realzar la adquisición de herramientas y mayor preparación para el trabajo — ha tendido a verse reducida a indicadores cuantitativos de los resultados en el empleo y también a expresarse en una simplificación de la transferencia de aprendizaje en prácticas y programas concretos.

Este fenómeno da cuenta de la invisibilización de su tratamiento especializado: el aporte interdisciplinario de la teoría de carrera con sus movimientos de educación para la carrera y orientación profesional, puesto que el mito racionalizado que - lo posiciona con estatus de regla general- fragmenta su puesta en acción respecto del propio quehacer organizacional de las universidades, las prácticas educativas, la investigación relacionada y las discusiones filosóficas acerca de su rol e ideas en torno a ella. Al tiempo en que persiste en políticas, reportes, estadísticas, sistemas de aseguramiento de la calidad y rankings internacionales con un manto de sentido común, que dificulta el estudio de este tipo de fenómenos al reproducir su aplicación problemática.

La empleabilidad —en el ámbito de la educación superiortiene origen, características e implicancias específicas que convocan a la investigación especializada a evitar su uso laxo y descontextualizado (como concepto, práctica e instrumento de política), por lo que requiere atender a un mayor diálogo interdisciplinar para el fortalecimiento de las bases de conocimiento propias. Con ello, se busca avanzar hacia la superación de falencias ya documentadas en la literatura y reducir aquellas asociadas a su aplicación en prácticas educativas concretas.

\section{Referencias}

Acosta, A. y Buendía, A. (2016). Perspectivas institucionales y educación superior desde miradas globales a espacios locales: el caso de México. Revista de la Educación Superior, 45(179), 9-23. http://dx.doi.org/10.1016/j.resu.2016.04.007

Álvarez, M. y Bisquerra, R. (2012). Orientación educativa. Modelos, áreas, estrategias y recursos. Madrid: Wolters Kluwer. 
Arora, B. (2013). A Gramscian analysis of the employability agenda. British Journal of Sociology of Education, 36(4), 635-648. http://dx.doi.org/10.1080/01425692.2013.838415

Artess, J., Hooley, T., \& Mellors-Bourne, R. (2017). Employability: A review of the literature 2012-2016. Recuperado de http://hdl.handle. net/10545/621285

Arulmani, G., Bakshi, A., Leong, F., \& Watts, A. (2014). Handbook of career development. International perspectives. New York: Springer International Publishing.

Barnett, R. (1990). The idea of higher education. Maidenhead: SRHE. Open University Press.

Barnett, R. (2000). Realizing the university. In an age of supercomplexity. Buckingham: SRHE. Open University Press.

Bastalich, W. (2010). Knowledge economy and research innovation. Studies in Higher Education, 35(7), 845-857.

http://dx.doi.org/10.1080/03075070903406533

Bell, D. (1976). The coming of post-industrial society. New York: Basic Books.

Ben-David, J. y Zloczower, A. (1962). La universidad en transformación. Barcelona: Seix Barral.

Bourdieu, P. (1997). Razones prácticas. Sobre la teoría de la acción. Barcelona: Anagrama.

Brown, B. \& Baker, S. (2007). Philosophies of research into higher education. London: Bloomsburry Publishers.

Brubacher, J. (1984). Filosofía de la enseñanza superior. Mexico D. F.: Editores Asociados Mexicanos.

Brunner, J. J. (2009). Educación superior en Chile. Instituciones mercados y politicas institucionales 1967-2007. Santiago de Chile: Universidad Diego Portales.

Burke, C., Scurry, T., Blenkinssop, J., \& Katy G. (2017). Critical perspectives on graduate employability. En M. Tomlinson \& L. Holmes (Eds.), Graduate employability in context. Theory, research and debate (pp. 87107). London: Palgrave Macmillan.

Coetzee, M. (2014). Psycho-social career meta-capacities. Dynamics of contemporary career development. Cham: Springer International Publishing.

Dacre Poll, L. \& Sewell, P. (2007). The key to employability: developing a practical model of graduate employability. Education and Training, 49(4), http://dx.doi.org/10.1108/00400910710754435 
Fairclough, N. (2003). Analyzing discourse: textual analysis for social research. London: Routledge.

Hager, P. (2006). Nature and development of generic attributes. En P. Hager $\&$ S. Holland (Eds.), Graduate attributes, learning and employability. Dordrecht: Springer International Publishing.

Hinchliffe, G. (2006). Graduate employability and lifelong learning: a need for realism? En P. Hager \& S. Holland (Eds.), Graduate attributes, learning and employability. Dordrecht: Springer International Publishing.

Holmes, L. (2013). Competing perspectives on graduate employability: possession, position or process? Studies in higher education, 38(4), 538554. http://dx.doi.org/10.1080/03075079.2011.587140

International Centre for Career Development and Public Policy, ICCDPP (2017). Career development at the crossroad communiqué 2017. Recuperado de http://iccdpp2017.org/download/Communique_2017_ ICCDPP.pdf

Kerr, C. (1962). The uses of the university. Harvard: Harvard University Press.

Knight, P. \& Yorke, M. (2003). Employability and good learning in higher Education. Teaching in Higher Education, 8(1), 3-16. https://doi.org/10.1080/1356251032000052294

Kogan, M. \& Brennan, J. (1993). Higher education and the world of work. Higher Education in Europe, 18(2), 2-23. https://doi.org/10.1080/0379772930180202

Krücken, G. (2011). A European perspective on new modes of university governance and actorhood. Recuperado de http://escholarship.org/ uc/item/54c9g6kp

Law, B. \& Watts, A. (1977). Schools, careers and community: Study of some approaches to careers education in schools. London: CIO Publishing for the GSBE.

Lyotard, J-F. (1984). The postmodern condition; a report on knowledge. Manchester: Manchester University Press.

Marginson, S. (2015). Rethinking education, work and 'employability'. Foundational problems of human capital theory. Recuperado de https://www.srhe.ac.uk/conference2015/downloads/Simon_ Marginson_Paper_SRHE2015\%20.pdf

McCarthy, J. (2016). La orientación profesional y las habilidades para la gestión de la carrera. Una perspectiva internacional. En A. Manzanares y C. Sanz (Eds.), Orientación profesional. Fundamentos y estrategias (pp. 49-64). Ciudad Real: UCLM. 
McQuaid, R. W. \& Lindsay, C. D. (2005). The concept of employability. Urban studies, 42(2), 197-219. http://dx.doi.org/10.1080/0042098042000316100

Meyer, J.W. \& Rowan, B. (1977). Institutionalized organizations: formal structures as myth and ceremony. American Journal of Sociology, 83(2), 440-463. https://doi.org/10.1086/226550

Newman, J. H. (1852/2016). La idea de una universidad. Santiago de Chile: Ediciones UC. (Trabajo original publicado en 1852).

Noguera, J. A. (2002). El concepto de trabajo y la teoría social crítica. Papers Revista de Sociología, 1(68), 141-168. http://dx.doi.org/10.5565/rev/papers/v68n0.1445

Oliveira, A. (2014). A history of the work concept from physics to economics. Dordrecht: Springer International Publishing.

Orellana, N. \& Serhati, J. (2017). Career development country report Chile, ICCDPP. Recuperado de http://iccdpp2017.org/download/Country_ paper_Chile_ENG.pdf

Pedersen, O. (1997). The first universities: Studium generale and the origins of university education in Europe. Cambridge: Cambridge University Press.

Rodríguez, M. L. (2002). Educación para la carrera y diseño curricular. Barcelona: Universitat de Barcelona.

Rothwell, A. \& Rothwell, F. (2017). Graduate employability: A critical oversigth. En M. Tomlinson \& L. Holmes (Eds.), Graduate employability in context. Theory, research and debate (pp. 41-63). London: Palgrave Macmillan.

Scott, P. (1984). The crisis of university. London: Croom Helm.

Scheele, J. y Brunner, J. J. (2009). Educación terciaria y mercado laboral: formación profesional, empleo, y empleabilidad. Revisión de la literatura internacional. Recuperado de http://www.udp.cl/funciones/ descargaArchivos.asp?seccion=documentos\&id=34

Shin, J. C. (2014). The scholarship of teaching, research, and service. En U. Teichler \& J. C. Shin, (Eds.), The future of the post-massified university at the crossroads restructuring systems and functions (pp. 75-84). Cham: Springer International Publishing.

Servicio de Información de Educación Superior, SIES (2018). Metodología buscador de empleabilidad e ingresos 2017-2018. Recuperado de http://www.mifuturo.cl/images/metodologias/metodologias_2018/ metodologia\%20buscador\%20empleabilidad\%20e\%20ingresos_2018. pdf 
Teichler, U. (2009). Higher education and the world of work. Conceptual frameworks, comparative perspectives, empirical findings. Rotterdam: Sense Publishers.

Teichler, U. (2015). Higher education and the world of work: The perennial controversial debate. En J. C. Shin, G. Postiglione, \& F. Huang (Eds.), Mass higher education development in East Asia (pp. 269-288). Cham: Springer International Publishing.

Tholen, G. (2013). What can research into graduate employability tell us about agency and structure? British Journal of Sociology of Education, 36(5), 766-784. http://dx.doi.org/10.1080/01425692.2013.847782

Tomlinson, M. (2017). Introduction: Graduate employability in context: Charting a complex, contested and multi-faceted policy and research field. En M. Tomlinson \& L. Holmes (Eds.), Graduate employability in context. Theory, research and debate (pp. 1-40). London: Palgrave Macmillan.

Trow, M. (1974). Problems in the transition from elite to mass higher education. Paris: OECD.

United Nations Educational, Scientific and Cultural Organization, Unesco (1998). Higher education for the twenty-first century vision and action. A European agenda for change for higher education in the Xl century. Recuperado de http://unesdoc.unesco.org/ images/0011/001133/113346Eo.pdf

Valiente, O. (2014). The OECD skills strategy and the education agenda for development. International. Journal of Educational Development, 39, 40-48. http://doi.org/10.1016/j.ijedudev.2014.08.008

Yorke, M. (2004). Employability in higher education: what it is - what it is not. Recuperado de https://www.qualityresearchinternational.com/ esecttools/esectpubs/Employability\%20in\%20HE.pdf

Zapata, G. y Clasing, P. (2016). El uso de criterios e indicadores de calidad en la acreditación de programas: diferencias entre agencias privadas de acreditación en Chile. Recuperado de https://www.cnachile.cl/ Biblioteca\%20Documentos\%20de\%20Interes/Cuaderno\%204_ZapataDigital.pdf 